\title{
NIVEL DE EFICACIA DE LA ACCIÓN TÉCNICO- TÁCTICA DEL TIRO A PORTERÍA EN EL FUTBOL.
}

Vera, Jose. ${ }^{1}$, Merchán, Rony. ${ }^{2}$, Jaimes, Marco. ${ }^{3}$

1. Doctor en ciencias de la cultura física, profesor universidad del valle, Grupo de Investigación Grinder. vera.jose@correounivalle.edu.com.co

2.Magister en Actividad Física, Docente Investigador Fundación Universitaria Juan de Castellanos. rdmerchan@jdc.edu.co

3. Doctor en nuevas perspectivas de investigación en la actividad física, Profesor Universidad de

Pamplona, mafrejala@hotmail.com Grupo de Investigación actividad Física y Deportes.

\section{RESUMEN}

El presente trabajo tuvo como finalidad Diseñar un Programa de entrenamiento para la acción técnico táctica del tiro a portería en el futbol.

Este estudio se enmarcó en una investigación de tipo mixto con tendencia cuantitativa y diseño descriptivo transversal, desarrollada con deportistas cuyas edades oscilan entre los 19 y los 24 años de los cuales 16 integraron la muestra.

La recolección de información se realizó a través de tres test específicos enfocados en las principales vías energéticas y enfocadas en la acción real de juego con finalización.

El procesamiento de datos se llevó a cabo con el programa estadístico SPSS, 22.0 fundamentando su análisis en una correlación de variables. Se concluyó que la aplicación de la metodología fundamentada en métodos integrales con finalización mejoro los resultados deportivos en los deportistas objeto de estudio y se logró construir un material que sirve de guía didáctica y pedagógica a los entrenadores y profesores de educación física de nuestra región.

Palabras clave: tiro a portería, futbol ofensiva, entrenamiento del fútbol

\section{LEVEL OF EFFECTIVENESS OF THE TECHNICAL-TACTICAL ACTION OF THE SHOOTING OF PORTERIA IN FOOTBALL.}

\begin{abstract}
This work aimed to establish a methodological approach to the training of technical-tactical action shot on goal in soccer.

This study was part of an investigation of mixed type with quantitative descriptive cross-sectional design trend and developed with athletes aged between 19 and 24 years of which 16 were part of the sample.

Data collection was performed through three specific tests focused on major energy pathways and focused on real action game completion.

Data processing was performed using SPSS statistical software 22.0 basing its analysis on a correlation of variables. It was concluded that the application of the methodology based on integral methods with completion improved athletic performance in athletes under study and managed to build a material that serves as a learning and teaching coaches and physical education teachers in our region guide.
\end{abstract}

Keyword: shot on goal, offensive football, soccer training 


\section{INTRODUCCIÓN}

El mundial de futbol de Brasil (2014) fue caracterizado como el mundial más ofensivo de la época contemporánea producto de una buena acción técnico- táctica del tiro a portería se pudo apreciar un nivel de eficacia de 171 goles todo esto es producto de la utilización del método global integrador ya que el mismo permite utilizar diferentes métodos competitivos cuyo producto final sea el tiro a portería.

En la actualidad se ha aumentado la publicación de diferentes trabajos científicos que nos permiten estudiar las diferentes tendencias del futbol y su evolución marcando un paso de métodos analíticos tradicionalistas a métodos integrales en donde la solución de problemas de pensamiento táctico y adecuadas tomas de decisiones es lo más utilizado.

Son varios los años que desde la academia hemos estudiado al futbol no solo desde el entrenamiento deportivo si no a través de la catedra universitaria se puede apoyar el trabajo del entrenador como antecedentes principales a esta investigación podemos citar diferentes trabajos realizados como por ejemplo (rivera, 2013) el libro de la acción técnico táctica del tiro a portería que aporta la creación y selección de diferentes juegos simplificados, pre-deportivos que se incluyen en la planificación especifica de la acción del tiro a portería en sus diferentes expresiones.

Otro trabajo importante a mencionar es la tesis de maestría de (Merchan, 2010). Titulada el nivel de eficacia técnica del jugador de futbol, este trabajo de investigación aporta unas pruebas específicas para los diferentes fundamentos técnicos que se caracterizan por aportar una tabla de valoración que permite apreciar el nivel de eficacia técnica del jugador de futbol tablas muy determinantes que utilizamos para el desarrollo de esta investigación a partir de estos dos importantes productos de investigación se han generado artículos cientíicos entre los más importantes pudiéramos referenciar los siguientes :

(Vera, 2008) Donde se justifica que es importante la elaboración de una metodología que a través de las teorías contemporánea los adelantos de la ciencia y la tecnología nos permitan mejorar el proceso de enseñanza y entrenamiento de los jugadores objeto de estudio a través de la Creación y selección de juegos simplificados y pre-deportivos y su inclusión en la planificación del entrenamiento deportivo, como se demuestra en la sesión de entrenamiento de las acciones técnico tácticas del tiro a portería.

Para el desarrollo de la metodología se utilizó la planificación del entrenamiento deportivo a través de direcciones y teniendo en cuenta las indicaciones metodológicas establecidas por (Forteza, 1997). En su libro "Alta metodología del entrenamiento deportivo" en donde realiza un estudio profundizado de las diferentes formas de planificar el proceso de entrenamiento.

En la medida que las investigaciones aumentaron, también los procesos de entrenamiento han tenido sus modificaciones. Actualmente son muchos los autores como por ejemplo (Garganta., 1996) , (Sans A, 2009), (Castaño, 2010), (Ibarra, 2010), (lopez, 2013), entre otras.

que sugieren que la naturaleza del fútbol es principalmente técnico táctica, afirmación que ha llevado a variar principalmente los contenidos de los entrenamientos y la planificación y dosificación de las cargas de trabajo.

La mayoría de las propuestas actuales se enfocan en los trabajos defensivos de los 
equipos de fútbol, priorizando la defensa zonal como la mejor manera de defenderse. Gran cantidad de libros, artículos, estudios e investigaciones del momento defensivo y de movimientos coordinados para neutralizar al rival muestran un panorama sesgado y limitado de este deporte debido a que el momento ofensivo se estudia en muy pocas investigaciones y libros. La fase ofensiva se muestra de una manera muy general, con conceptos tácticos definidos teóricamente, pero muy pocas valoraciones de dosificación, control, planificación y jerarquización de una serie de principios tácticos necesarios para tratar de contrarrestar los movimientos defensivos de los rivales.

En el trabajo de la fase ofensiva es indispensable hacer mayor cantidad de goles que el rival, como lo establece el reglamento. Teniendo en cuenta esto, la búsqueda del gol se vuelve fundamental para obtener la victoria, por lo que se debe prestar atención en la búsqueda de métodos, movimientos y estrategias para romper los sistemas defensivos que imponen los rivales.

Se hace necesario definir una idea de juego en el momento de ataque, para posteriormente a través de la planificación buscar una serie de patrones ofensivos del equipo para tratar de lograr la mayor cantidad de goles posibles,y es ahí donde a través de este trabajo se busca sugerir ideas, aportes de conceptos y movimientos que puedan facilitar vulnerar defensas zonales presionantes aplicadas en poco espacio y con una gran cantidad de jugadores por parte de los rivales, y preferiblemente en campo propio.

Desde el punto de vista del entrenamiento integral, involucrando los aspectos técnicos tácticos, psicológicos y físicos.

(Alfano, 2011)propone y recomienda el trabajo intermitente como el tipo de ejercicio específico para futbolistas a traves de la utilizacion de los componenetes de la carga que se integren a situacion de juego que permitan el transito de defensa a ataque.

Otro estudio de tipo interdisciplinario que ayudaría a esta investigación de forma general son los resultados publicados por (barajas, 2005) en cuanto a la importancia de estudio biomecánico del tiro a portería, este articulo aporta a nuestra investigación la importancia que tiene el análisis de los movimientos ofensivos sobre todo cuando estamos buscando una efectividad para llegar al rompimiento continuo de los trabajos zonales de las defensas del contrario. Acero y Barajas (2005) en su tesis magistral efectividad de un programa individualizado de optimización de la técnica (PIOT) basado en un análisis antropocinemático del tiro libre preferencial (TLP) en jugadores de la selección de fútbol de la Universidad de Pamplona, Colombia, plantean lo siguiente: La metodología de la investigación incluye la evaluación biomecánica inicial de la técnica del tiro libre a portería en el fútbol utilizando el software SC.PRO v.5 para análisis biomecánicos bidimensionales (2D y 3D) del Instituto de Investigación \& Soluciones Biomecánicas. Cali, Colombia.

En la mayoría de los libros y las investigaciones se plantea aspectos muy importantes sobre todo de carácter general por ejemplo (lopez, 2013) en su libro escribe de forma metodológica general aportando a nuestra investigación una muy buena caracterización de cómo debe abordarse el entrenamiento tatico ofensivo y defensivo , se aprecian algunos ejercicios de forma general pero se desconoce la dosificación de carga que se le deben de dar a los mismos, los principios tácticos ofensivos y la importancia de su entrenamiento, pero con poca claridad en la metodología de entrenamiento, control de las cargas y test para su análisis.

(lopez, 2013) En su libro ataque combinado en un sistema 1-4-3-3, organización y tareas de entrenamiento, se acerca mucho a nuestra propuesta, ya que 
en este documento muestra la fase ofensiva de un equipo en la fase de iniciación (desde portería propia), ante un rival que presiona fuertemente en posición avanzada y también las posiciones que deben tomar los jugadores ante un rival que presiona en zona intermedia.

(Alonso, 2012) En su libro entrenamiento de la táctica ofensiva volumen 2, presenta una serie de ejercicios de acuerdo a principios tácticos ofensivos que el mismo autor define y considera fundamentales en la fase ofensiva de un equipo de fútbol, y los demuestra a través de la siguiente clasificación:

- Sesiones de entrenamiento para la mejora de la eficacia en la progresión del ataque en un juego predominante de bandas.

- Sesiones de entrenamiento sobre la posesión del balón para la mejora de los ataques combinativos.

- Sesiones de entrenamiento para la mejora de la posesión del balón.

- Sesiones de entrenamiento para la mejora de la amplitud y profundidad ofensiva.

- Sesiones de entrenamiento para la mejora de los cambios de orientación.

- Sesiones de entrenamiento para la mejora de la posesión del balón, amplitud ofensiva y los cambios de orientación en los ataques combinativos.

(Rozen, 2008) en su libro, táctica, metodología del gol, enfoca su análisis en los conceptos del fútbol, en sus fases defensivas y ofensivas, y habla de un deporte de constantes transiciones defensivas y ofensivas que se repiten constantemente y que son fundamentales para el equilibrio tanto defensivo como ofensivo.

En la fase ofensiva, y teniendo presente que el fin prioritario es el logro de goles, los jugadores cuidarán fundamentalmente de, jugar tanto a lo ancho (amplitud) como a lo largo (profundidad/ penetración), alternando el juego sobre el eje transversal y longitudinal del campo de juego; mantener la posesión del balón y de esta forma intentar vulnerar al adversario. Recomienda después de recuperar el balón, tratar de mantener la posesión a través de pases horizontales, verticales, diagonales tanto a ras de piso como aéreos y también a través de habilidades individuales.

Teniendo en cuenta estas orientaciones y las características del entrenamiento del fútbol como el objetivo planteado; se elaboró un plan de entrenamiento a través de modelos de mesociclos, microciclos y sesiones de entrenamiento.

\section{Objetivo:}

- Diseñar un Programa de entrenamiento para la acción técnico táctica del tiro a portería en el futbol.

\section{METODOLOGÍA Y PROCEDIMIENTO.}

Para (Hernandez, 2010 ). "Los métodos de investigación mixta son la integración sistemática de los métodos cuantitativo y cualitativo en un solo estudio con el fin de obtener una "fotografía" más completa del fenómeno", teniendo en cuenta esta definición y las particularidades del presente trabajo, se define que el tipo de investigación desarrollada fue mixta con tendencia cuantitativa, toda vez que los datos recolectados a través de diferentes herramientas cualitativas y cuantitivas arrojaron resultados que fueron sometidos a un análisis estadístico que apuntó a la caracterización de la muestra y la correlación de variables.

Teniendo en cuenta las carecteríaticas que enmarcan esta investigación, los objetivos que persigue y las herramientas empleadas 
durante su desarrollo, se puede concluir que su diseño es descriptivo transversal, según (Mendoza, 2004) este tipo de diseño busca "describir las características que se estudian de los sujetos que integran la muestra seleccionada ... el procedimiento consiste en medir, observar o aplicar entrevistas y/o encuestas, según lo que se estudia a un grupo de personas y proceder a su descripción".

Esta caracterización del proceso deportivo se puede apreciar en el siguiente esquema que resume las diferentes fases de la investigación en donde a partir de la especificidad del objeto de estudio se da a conocer como aporte el siguiente programa de entrenamiento con profundización en la acción técnico - táctica de la acción técnico táctica del tiro a portería.

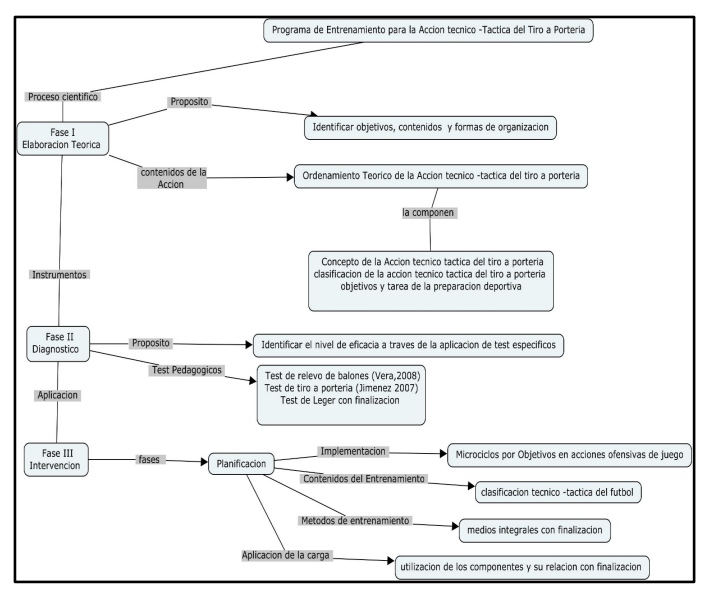

Figura 1. Esquema para el programa de entrenamiento de la Acción técnico táctica del tiro a portería. Fuente: los autores

El esquema planteado busca contribuir al crecimiento teórico y práctico del entrenamiento táctico ofensivo con acentuación en la acción técnico- táctica del tiro a portería, favoreciendo la consolidación de la ofensiva en el fútbol como disciplina del entrenamiento deportivo que sirve como una guía teórica, a las personas interesadas en este deporte (jugadores, entrenadores, preparadores físicos, etc.).
De acuerdo a lo planteado en la figura 1 , Programa para el entrenamiento de la accion tecnico- tactica del tiro a porteria diseñado en el proceso investigativo, este comprende tres fases:

La primera fase identifica objetivos que van directamente a perfeccionar el rendimiento deportivo a partir del ordenamiento teorico de la accion tecnico -tactica del tiro a porteria, que tiene como finalidad la anotacion del gol a traves de la culminacion de una jugada ofensiva.

La fase II se aplico con el objetivo de identificar y aplicar pruebas especificas que nos permitan cuantificar de forma confiable la accion tecnico -tactica del tiro a porteria.

Los test aplicados como instrumentos pedagogicos del entrenamioento deportivo fueron los siguientes:

Test de relevos de balones. (Vera, Metodologia del entrenamiento de la accion tecnico tactica del tiro a porteria en el futbol infantil , 2008) tiene como objetivo evaluar las acciones tecnico-tacticas de la finalizacion teniendo encenta una intensidad maxima a la hora de realizar el test.

Test de tiro a porteria. (Jimenez, 2002) este test mide el nivel de eficacia del tiro a porteria a traves de 20 intentos en cuatro direcciones y se evalua a traves de una tabla de valoracion por puntos que describen el nivel de eficacia que el jugador alcanza.

Test de leger este tradicional test tiene como objetivo cuantificar la resistencia especifica del jugador de futbol.

La fase III. lo conforma la intervención pedagógica esta se resume en la utilización de modelos de mesociclos que se diseñan a partir de las tendencias contemporáneas de la planificación del entrenamiento deportivo en donde a partir de lo estudiado se dosifico la preparación deportiva a través de ciclos pequeños y para este trabajo se utilizó los contenidos que componen la acción técnico - táctica del tiro a portería por lo que se diseñó una estructura pedagógica a través de los métodos de entrenamiento que se relacionan con los componentes de la carga 
a través de la utilización de series, repeticiones, volúmenes e intensidades en los ejercicios variables, invariables y competitivos siempre con finalización a continuación podemos apreciar algunos ejemplos prácticos de juegos globales integradores con tiro a portería.

Juego 1. Zona de trabajo y recuperación activa.

\section{Descripción metodológica:}

1. para la zona de trabajo.

Cuatro participantes por equipo, utilizamos conos o platillos y varios arcos móviles al no tenerlos remplazar las portería utilizando conos grandes, cada uno con un balón y al frente de una formación de obstáculos (conos o platillos) a la orden del profesor van al primer cono a máxima velocidad conduciendo el balón se devuelven al punto de partida se dirigen al segundo cono y así sucesivamente hasta llegar al final del recorrido el cual termina con lanzamiento a puerta e inmediatamente después van a buscar un tercer balón y ejecutan en el espacio restante un enfrentamiento de cuatro contra cuatro.

2. para la zona recuperación.

En esta zona jugamos voleo-fútbol con tiro a puerta. El juego consiste en que el arquero realiza un saque por encima de la malla a su compañero el cual deja picar una vez y empieza a pasarlo a los demás sin dejar caer el balón debe pasar por los 4 miembros del equipo y el último es quien realiza tiro a puerta tratando de anotar. A la pérdida del balón, saca el equipo contrario y así sucesivamente.

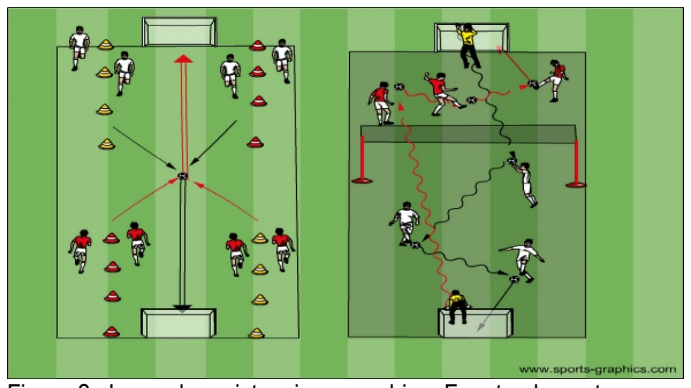

Figura 2. Juego de resistencia anaerobica. Fuente : los autores
Sitema de carga para el entrenamiento de la resistencia anaerobica

Descripcion de la tabla : a traves del metodo de repeticiones uniforme realizar las series y repeticiones descritas en la siguiente tabla.

\begin{tabular}{|l|l|l|l|l|}
\hline Series & Tandas & $\begin{array}{l}\text { Tiempo } \\
\text { en } \\
\text { zona } \\
\text { de } \\
\text { trabajo }\end{array}$ & $\begin{array}{l}\text { Tiempo en } \\
\text { zona de } \\
\text { recuperación }\end{array}$ & $\begin{array}{l}\% \text { de } \\
\text { intensidad }\end{array}$ \\
\hline 1 & 1 & $2 \mathrm{~min}$ & $3 \mathrm{~min}$ & $80 \%$ \\
\hline & 2 & $2 \mathrm{~min}$ & $3 \mathrm{~min}$ & $80 \%$ \\
\hline & 3 & $2 \mathrm{~min}$ & $3 \mathrm{~min}$ & $80 \%$ \\
\hline & 4 & $2 \mathrm{~min}$ & $3 \mathrm{~min}$ & $80 \%$ \\
\hline
\end{tabular}

Tabla 1. Sitema de carga para el entrenamiento de la resistencia anaerobica

Resistencia aerobica

Descripción metodológica:

Se forman de a 4 alrededor de la cancha, identificando 2 vs 2 cada jugador en posesión del balón cumplirán las siguientes indicaciones metodológicas.

Realizan carrera a diferentes ritmos moderados y darán respuesta a la indicación metodológica del profesor el cual asignara como ejemplo realizar juegos simplificados o tiro a portería tratando de mantener una intensidad suave y moderada

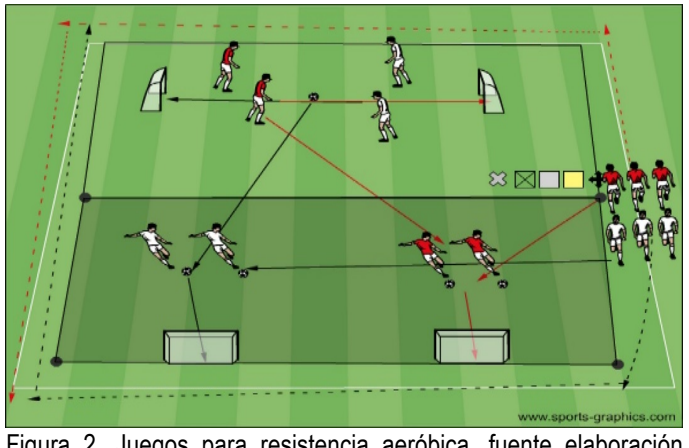

Figura 2. Juegos para resistencia aeróbica, fuente elaboración propia.

Acciones simplificadas de juego.

Descripción metodológica: Dos equipos de igual número de participantes enumerados individualmente, el profesor es quien da la indicación metodológica de que jugador 
patea y como lo hace. El equipo ganador es aquel que consiga anotar más goles. Además debe realizar enfrentamientos de 2 vs 2,3 vs 3 etc....

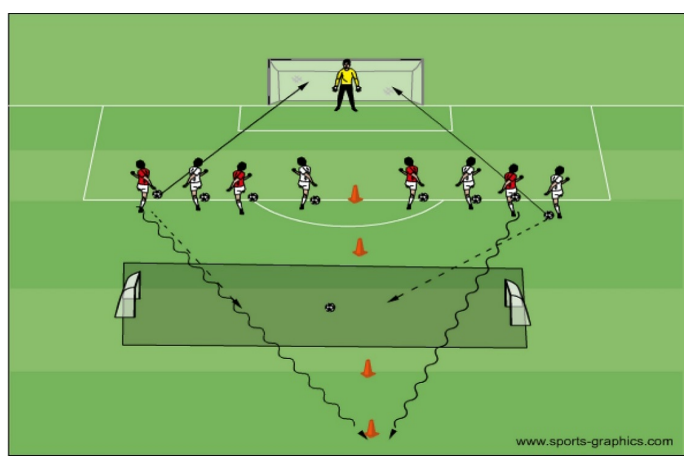

Figura 3. Tiro a portería en acción simplificada de juego. Fuente los autores.

\section{RESULTADOS Y DISCUSIÓN GENERAL.}

En esta fase se analizó la información recolectada en torno a las variables de la investigación, referente a la comparación entre el grupo control conformado por jugadores a los mismo se le aplico entrenamiento tradicional de una forma empírica sin necesidad de llevar planificación ni control del proceso de entrenamiento y sin profundizar en la acción técnico- táctica del tiro a portería manteniendo una frecuencia 6 de entrenamiento, participando en las mismas competencia y participando con las mismas normas y las mismas pruebas del pre-test y pos-test.

Y el grupo experimental se le aplico la propuesta fundamentada en una planificación enfocada a la utilización de métodos y medios que siempre estaban acompañados de la acción del tiro a portería.

Las variables a medir fueron tiro a portería en resistencia anaeróbica, nivel de eficacia del tiro a portería y resistencia aeróbica todo esto para cumplir con el desarrollo de los objetivos específicos que requieren de instrumentos de medición y así realizar comparaciones de resultados entre el pre-test el post-test y entre grupos a través de técnicas estadísticas como el estudio de la probabilidad y así comprobar a través de $p$-valor si hubo diferencias significativas entre el pre-test y el pos test para poder determinar la eficacia de la acción técnico- táctica del tiro a portería ; el nivel de significancia fue del $5 \%$. También este análisis se realizó mediante la utilización de estadística no paramétrica dado que no necesariamente deben cumplir supuestos. Para analizar los datos se utilizó el SPSS (paquete estadístico para las ciencias sociales) el Statistix $22.0 \mathrm{y}$ el Excel, de este análisis los resultados más importantes fueron los siguientes:

El $90 \%$ del equipo mejoró su resistencia anaeróbica en combinación con las acciones más importantes de la ofensiva que fueron el tiro a puerta su efectividad, y la conducción del balón mejorando casi totalmente los resultados del pre-test.

Aplicada la prueba $T$ Students observamos en la prueba Tiro a portería que en el Pre test se encontraron diferencias muy significativas del grupo Experimental sobre el Control registrándose un valor $\mathrm{P}<0.0073$. Para el Post luego de la intervención con entrenamiento de metodología global y metodología analítica con ejercicios de finalización encontramos estadísticamente diferencias altamente significativas en el Grupo Experimental vs el Grupo Control registrándose un valor $P<0.0000$. Los promedios de del pre y post en los dos grupos son relativamente diferentes, siendo notorios los resultados en el Experimental. En cuanto la relación existente entre el nivel de eficacia técnica en el grupo experimental tanto en el pre test como en el post test se encuentra en un nivel de evaluación Bueno ahora el grupo control en el pre test su relación con el nivel de eficacia se encuentra en un nivel de evaluación insuficiente, pero en el post test este grupo asciende a un nivel aceptable.

En cuanto al test de leger Los porteros se encuentran muy por debajo del rango normal que deberían tener, La mayoría se 
encuentra en el rango de regular- bien (ubicados entre 50 a $60 \mathrm{ml} / \mathrm{kg} / \mathrm{min}$, datos normales en cuanto al vo2 máximo promedio en jugadores de fútbol, 7 jugadores se encuentran entre 46 y 48 $\mathrm{ml} / \mathrm{kg} / \mathrm{min}$, lo que los ubica en un rango algo bajo en su resistencia física, solo dos jugadores están por encima del promedio, marcando un dato bastante elevado con respecto a los demás compañeros.

\section{REFERENCIAS BIBLIOGRÁFICAS}

Barajas, J. A. (2005). biomecanica bidimensional (2d) del tiro libre preferencial en el futbol : modelo integral exprimental . pamplona : tesis de maestria universidad de pamplona .

Castaño, J. (2010). Incidencia de un plan de entrenamiento basado en el metodo global y la planificacion tactica, en la fase ofensiva y defensiva del equipo juvenil del club deportivo barrio brasilia . medellin: tesis de grado universidad de atioquia .

Forteza, A. (1997). Alta Metodologia del Entrenamiento Deportivo . Madrid : gynos .

Garganta. (1996). Modelo Tactico de juego de futbol : estudio organizacion de la fase ofensiva de equipos de alto rendimiento. Porto: Tesis doctoral Universidad de porto.

Hernandez, F. y. (2010). metodologia de la investigacion cientific . mexico : Mc Graw Hill.

Ibarra, O. G. (2010). incidencia de un plan de entrenamiento fundamentado en el principio tactico ofensivo de la conservacion del balon en futbolistas juveniles del club de formacion deportiva nororiental . medellin : tesis de grado universidad de antioquia .

Jimenez. (2002). Evaluacion tecnica $y$ tactica del futbol . medellin : Trabajo de investigacion de la universidad de antioquia

Lopez, J. L. (2013). Bases teorico practicas del entrenamiento especifico del futbol. Madrid : wanceulen.

Mendoza, M. A. (2004). La Investigacion cientifica en la actividad fisica : su metodologia . La habana : Deportes .

Merchan, R. (2010). Nivel de eficacia tecnica del jugador de futbol base. Pamplona : trabajos de investigacion de maestria en ciencias de la actividad fisica y deporte universidad de pamplona .

Vera, j. o. (2013). La accion tecnico- tactica del tiro a porteria en el futbol infantil. Berlin: Academica Española. 